\title{
ESTIMATIONS OF THE LENGTHS AND PERIODS OF CLOSED TRAJECTORIES*
}

By K. R. SCHNEIDER (Deutschen Akademie der Wissenschaften zu Berlin)

Let $\Gamma$ be a closed trajectory, of period $\omega$, of the two-dimensional autonomous system of differential equations

$$
d x / d t=P(x, y), \quad d y / d t=Q(x, y),
$$

where $P(x, y)$ and $Q(x, y)$ belong to class $C^{1}$ for all $x$ and $y$. Let $B$ be a region of finite area $|B|$, free of singular points and containing in its interior the region $A$, in which lies the closed trajectory $\Gamma$. Under these assumptions S. P. Diliberto [1] has given bounds for $\omega$. This bound depends solely on norm $\|X\|$ of the vector-function $X(x, y) \equiv\{P(x, y)$, $Q(x, y)\}$, curvature $k(x, y)$ of the trajectory of system (1) passing through $(x, y)$, curvature $h(x, y)$ of the orthogonal trajectory and some measure of the boundness properties of $A$.

In a recent paper P. J. Lau [2] deals with the same problem. By strengthening the suppositions on the boundaries of $A$ he is able to define $B$ as a $q$-parallel ring $A_{q}$ of $A$, where $q$ depends on curvatures $k(x, y)$ and $h(x, y)$, where $(x, y) \in G \supset A_{q}$. His bound for the period $\omega$, which is an improvement of the bound given by Diliberto [1], again depends on $\|X\|$, curvatures $k(x, y)$ and $h(x, y)$ and area of the $q$-parallel ring $A_{a}$. In this paper we shall give a method to obtain upper and, generally, even nontrivial lower bounds for the lengths and periods of periodic solutions of (1) lying in a bounded doubly connected region $A$. Often the results obtained will be better than those obtained by the method of Lau [2]. The upper and lower bounds of $\omega$ obtained by our method are solely functions of $\|X\|$ on $A$, of area $A$ and of $k(x, y)$ on $A_{e}$, where $A_{\bullet}$ denote the simply connected region which is bounded by the exterior boundary $\partial A_{e}$ of $A$.

Let $A_{\Gamma}$ denote the region bounded by the closed trajectory $\Gamma$. Suppose that the functions $P(x, y)$ and $Q(x, y)$ belong to class $C^{1}$ for all $(x, y) \in \widetilde{A} \supset A_{\Gamma}$ and that the number of singular points of the system (1) is finite in $\tilde{A}$. We state the following theorem:

THEOREM.

$$
\left|\iint_{A_{\Gamma}} k(x, y) d x d y\right|=L
$$

where $L$ denotes the length of $\Gamma$.

Proof. First we remark that the curvature

$$
k(x, y) \equiv \frac{P^{2} Q_{x}+P Q\left(Q_{y}-P_{x}\right)-Q^{2} P_{y}}{\left(P^{2}+Q^{2}\right)^{3 / 2}}
$$

may be written as

${ }^{*}$ Received September 6, 1968. 


$$
k(x, y) \equiv \frac{\partial}{\partial x}\left[Q\left(Q^{2}+P^{2}\right)^{-1 / 2}\right]+\frac{\partial}{\partial y}\left[-P\left(Q^{2}+P^{2}\right)^{-1 / 2}\right] \equiv \operatorname{div} \mathbf{a},
$$

where

$$
\mathbf{a} \equiv\left\{Q\left(Q^{2}+P^{2}\right)^{-1 / 2},-P\left(Q^{2}+P^{2}\right)^{-1 / 2}\right\} .
$$

Let $S_{i}, i=1,2, \cdots, n$, denote the singular points of $(1)$ in $A_{\Gamma}$ and $K_{t}\left(S_{i}\right)$ the circle with center $S_{i}$ and radius $\epsilon$. Let $\epsilon$ be sufficiently small that the relation $\bigcup_{i=1}^{n} K_{\epsilon}\left(S_{i}\right) \cap$ $\partial A_{\Gamma}=\varnothing$ is valid ( $\varnothing$ : empty set, $\partial A_{\Gamma}$ : boundary of $A_{\Gamma}$ ). Thus in the region $A_{\Gamma} \backslash \bigcup_{i=1}^{n} K_{\epsilon}\left(S_{i}\right)$ all suppositions of the Gaussian theorem are fulfilled. Applying this theorem and (2) we obtain

$$
\begin{aligned}
& \iint_{\mathcal{A} \Gamma \underbrace{n}_{i=1} K_{e}\left(S_{i}\right)} k(x, y) d x d y=\iint_{\mathcal{A} \Gamma \backslash \underbrace{n}_{i=1} K_{e}\left(S_{i}\right)} \operatorname{div} \mathbf{a} d x d y \\
& =\oint_{\Gamma} \mathbf{a} \cdot \mathbf{n}_{\Gamma} d s+\sum_{i=1}^{n} \oint_{K_{e}\left(s_{i}\right)} \mathbf{a} \cdot \mathbf{n}_{\mathbf{i}} d s,
\end{aligned}
$$

where $\mathbf{n}_{\Gamma}$ and $\mathbf{n}_{i}$ denote the unit vectors in the direction of the outward normals of $A_{\Gamma}$ and $K_{\epsilon}\left(A_{i}\right)$ respectively.

According to the definition of a, the following relation is valid:

$$
\mathbf{a} \cdot \mathbf{n}_{i}=\|\mathbf{a}\|\left\|\mathbf{n}_{i}\right\| \cos \left(\mathbf{a}, \mathbf{n}_{i}\right)=\cos \left(\mathbf{a}, \mathbf{n}_{i}\right) \text {. }
$$

Thus, because of $\left|\cos \left(\mathbf{a}, \mathbf{n}_{i}\right)\right| \leq 1$, we have

$$
\begin{array}{r}
\lim _{\epsilon \rightarrow 0}\left|\sum_{i=1}^{n} \oint_{K_{e}\left(S_{i}\right)} \mathbf{a} \cdot \mathbf{n}_{i} d s\right|=\lim _{\epsilon \rightarrow 0}\left|\sum_{i=1}^{n} \oint_{K_{e}\left(S_{i}\right)} \cos \left(\mathbf{a}, \mathbf{n}_{i}\right) d s\right| \\
\leq \lim _{\epsilon \rightarrow 0} \sum_{i=1}^{n}\left|\oint_{K_{e}\left(S_{i}\right)} d s\right|=\lim _{\epsilon \rightarrow 0} 2 \pi n \cdot \boldsymbol{\epsilon}=0 .
\end{array}
$$

Therefore we obtain from (4)

$$
\iint_{\boldsymbol{A}_{\mathbf{\Gamma}}} k(x, y) d x d y=\oint_{\Gamma} \mathbf{a} \cdot \mathbf{n}_{\Gamma} d s .
$$

Because $\Gamma$ is a closed trajectory of the system (1) and $n_{\Gamma}$ is the unit vector in the direction of the outward normal of $A_{\Gamma}$, we have $\mathbf{a} \cdot \mathbf{n}= \pm 1$. Consequently, we obtain from (5)

$$
\left|\iint_{A_{\Gamma}} k(x, y) d x d y\right|=\left|\oint_{\Gamma} d s\right|=L .
$$

This theorem provides us the possibility of estimating the length $L$ and the period $\omega$ of $\Gamma$.

Let $A$ be a doubly connected region containing the closed trajectory $\Gamma$. We suppose that the interior boundary $\partial A_{i}$ and the exterior boundary $\partial A_{e}$ of $A$, bounding the simply connected regions $A_{i}$ and $A_{e}$, are composed of a finite number of smooth arcs. In addition we assume that $A$ is free of singular points of the system (1) and $A_{\varepsilon} \subset \widetilde{A}$.

Using the notations

$$
k=\max _{(x, y) \in A}|k(x, y)|, \quad|A|=\operatorname{area} A
$$


we estimate

$$
\begin{aligned}
L & =\left|\iint_{A_{\Gamma}} k(x, y) d x d y\right| \leq\left|\iint_{A_{i}} k(x, y) d x d y+\iint_{A_{\Gamma} \backslash A_{i}} k(x, y) d x d y\right| \\
& <\left|\iint_{A_{i}} k(x, y) d x d y\right|+k|A|
\end{aligned}
$$

and

$$
L \leq\left|\iint_{A_{e}} k(x, y) d x d y-\iint_{A_{e} \backslash A_{\Gamma}} k(x, y) d x d y\right|<\left|\iint_{A_{e}} k(x, y) d x d y\right|+k|A|
$$

Analogously, we can obtain a lower bound for $L$. Consequently, the following corollary is valid:

\section{Corollary 1.}

$L<L_{2}=\min \left\{\left|\iint_{A_{i}} k(x, y) d x d y\right|,\left|\iint_{A_{e}} k(x, y) d x d y\right|\right\}+k|A|$,

$L>\left\{\begin{array}{l}L_{1}=\max \left\{\left|\iint_{A_{i}} k(x, y) d x d y\right|,\left|\iint_{A_{e}} k(x, y) d x d y\right|\right\}-k|A|, \\ \left|\partial A_{i}\right| \text { otherwise. }\end{array}\right.$

If $k(x, y)$ possesses the same sign in $A_{e} \backslash \bigcup_{i=1}^{n} S_{i}$, we have

$$
\begin{aligned}
\bar{L}_{1} & =\min \left\{\left|\iint_{A_{i}} k(x, y) d x d y\right|,\left|\iint_{A_{e}} k(x, y) d x d y\right|\right\}<L \\
& <\max \left\{\left|\iint_{A_{i}} k(x, y) d x d y\right|,\left|\iint_{A_{e}} k(x, y) d x d y\right|\right\}=\bar{L}_{2}
\end{aligned}
$$

Let be $s$ the arc length on $\Gamma$. By means of the notations

$$
\begin{aligned}
& m_{i}=\min _{(x, y) \in A}\left\{[P(x, y)]^{2}+[Q(x, y)]^{2}\right\}^{1 / 2}=\min _{(x, y) \in A}\|X(x, y)\|, \\
& m_{a}=\max _{(x, y) \in A}\|X(x, y)\|
\end{aligned}
$$

we obtain from relation

$$
|d s / d t|=\|X(x, y)\|_{1 \mathbf{r}}
$$

Corollaky 2. For the period $\omega$ of the closed trajectory, the following estimations are valid:

$\omega<\frac{1}{m_{i}}\left[\min \left\{\left|\iint_{A_{i}} k(x, y) d x d y\right|,\left|\iint_{A_{e}} k(x, y) d x d y\right|\right\}+k|A|\right]$,

$\omega>\left\{\begin{array}{l}\frac{1}{m_{a}}\left[\max \left\{\left|\iint_{A_{i}} k(x, y) d x d y\right|,\left|\iint_{A_{e}} k(x, y) d x d y\right|\right\}-k|A|\right], \\ \quad \text { if this expression is greater than }\left|\partial A_{i}\right| m_{a}^{-1}, \\ \left|\partial A_{i}\right| m_{a}^{-1} \quad \text { otherwise. }\end{array}\right.$ 
If $k(x, y)$ posseses the same sign in $A_{e} \backslash \bigcup_{i=1}^{n} S_{i}$, we have

$$
\frac{1}{m_{a}} \cdot \bar{L}_{1}<\omega<\frac{1}{m_{i}} \cdot \bar{L}_{2} \text {. }
$$

For illustrating our results we first consider the very simple system

$$
d x / d t=-y, \quad d y / d t=x .
$$

We wish to obtain estimations of the length $L$ and of the period $\omega$ of the closed trajectory $\Gamma$ passing through the point $x=0, y=1$. It is known that the relations $L=2 \pi$ and $\omega=2 \pi$ are valid.

Let us suppose that the region $A$ containing the closed trajectory $\Gamma$ is represented by the region which is bounded by the circles $C_{1}: x^{2}+y^{2}=(0.9)^{2}$ and $C_{2}: x^{2}+y^{2}=(1.1)^{2}$. Since $k(x, y)=\left(x^{2}+y^{2}\right)^{-1 / 2}$ possesses the same sign in $A_{e} \backslash\{0\}$, we obtain from Corollary 1 the estimation $2 \pi \cdot(0.9) /(1.1) \leq \omega \leq 2 \pi \cdot(1.1) /(0.9)$. The application of the method of Lau [2] yields $L \leq 2 \pi \cdot(1+(0.1) / p)=\tilde{L}_{2}$ with $p<0.9$. A comparison of $\bar{L}_{2}$ and $L_{2}$ demonstrates $\bar{L}_{2}<\tilde{L}_{2}$. As a second example we consider the system

$$
d x / d t=-y+x\left[\left(x^{2}+y^{2}\right)^{1 / 2}-1\right], \quad d y / d t=x+y\left[\left(x^{2}+y^{2}\right)^{1 / 2}-1\right] .
$$

This system posseses only one nontrivial periodic solution. The corresponding closed trajectory $\Gamma$ is represented by the circle $x^{2}+y^{2}=1$ with $L=2 \pi$ and $\omega=2 \pi$.

Let us again suppose that the region $A$ containing $\Gamma$ is formed by the region bounded by $C_{1}$ and $C_{2}$. Since the function $a(r)$,

$$
a(r)=\iint_{A r} k(x, y) d x d y=\iint_{A r} \frac{2-\left(x^{2}+y^{2}\right)}{\left(1+\left[\left(x^{2}+y^{2}\right]^{1 / 2}-1\right)^{2}\right)^{3 / 2}} d x d y=\frac{2 \pi r}{\left[1+(r-1)^{2}\right]^{1 / 2}}
$$

where $A_{r}$ is the simply connected region bounded by the circle $x^{2}+y^{2}=r^{2}$, is monotone, increasing for $0.9 \leq r \leq 1.1$, we have the estimations

$$
\begin{gathered}
2 \pi \cdot 0.9 \leq(1.01)^{1 / 2} \cdot L \leq 2 \pi \cdot 1.1=\bar{L}_{2} \cdot(1.01)^{1 / 2} . \\
2 \pi \cdot \frac{0.9}{1.1} \leq 1.01 \omega \leq 2 \pi \cdot \frac{1.1}{0.9} .
\end{gathered}
$$

If we use the method of Lau we obtain $L \leq 2 \pi\left(1+0.1 \cdot p^{-1}\right)=I_{2}, p<0.9$. A comparison again yields $\bar{L}_{2}<\tilde{L}_{2}$.

\section{ReFERENCES}

[1] S. P. Diliberto, "Bounds for periods of periodic solutions" in Contributions to the theory of nonlinear oscillations, Vol. III, Annals of Math. Studies, no. 36, Princeton Univ. Press, Princeton, N. J., 1955, pp. 269-275

[2] P. J. Lall, Bounds for the lengths and periods of closed orbits of two-dimensional autonomous systems of differential equations, J. Differential Eqs. 3, 330-342 (1967) 